\title{
Hair Restoration in Cranial Surgery Patients
}

\author{
Parsa Mohebi, MD, FISHRS II Los Angeles, California, USA I info@parsamohebi.com; William R. Rassman, MD I \\ Los Angeles, California, USA
}

Scalp scars can be a huge burden in patients who already have associated and complicated health challenges. For the neurosurgical patient, scalp incisions following cranial surgery may result in unique aesthetic problems. For example, transected hair follicles can cause hair loss in surrounding areas. In addition, scalp scars often stretch when incisions are not made parallel to Langer's lines (aka relaxed skin tension lines).

Shaving the head before neurosurgical procedures has been a matter of debate for years due to its psychosocial disruption, and several studies were performed to address this issue. ${ }^{1-5}$ However, scars from neurosurgical surgeries can produce an even bigger and more permanent problem for this group of patients. Most neurosurgical scars or other cranial scars are surrounded by normal hair, which does offer a degree of camouflage. A problem arises when there is not enough native hair to cover the scar (particularly in men with male pattern hair loss [MPHL]) or when the scar extends beyond the areas of normal hair distribution. Location, direction, and the method of wound closure can affect the visibility of the final neurosurgical scars.

A number of techniques have been recommended to improve scalp scars. De-epithelialization of one or both wound edges, trichophytic closure, allows hair to grow into the scalp scar and make it less visible. ${ }^{6}$ Also, zigzag incisions have been used to reduce scar visibility with some success. ${ }^{7,8}$ More recently, scalp micropigmentation (SMP) techniques have been developed as a non-surgical cosmetic solution to scars. ${ }^{9}$

The appearance of an undesirable scar is inevitable in many circumstances regardless of the surgical technique. The final scalp scars could be disfiguring and hair transplantation might be desirable to disguise them. Follicular unit transplantation (FUT) can produce natural looking results that can match a normal head of hair in patients with MPHL. ${ }^{10}$ This technique can also be used to fill in a scalp scar by transplanting directly into it. Due to the diversity of neurosurgical scars and their needs, different hair restoration methods are used to camouflage them.

Neurosurgical scars produce disfigurement primarily in two ways. One is when the scalp scar is in a dense hair-bearing area of scalp so a lack of hair in the scar in comparison to surrounding areas produces a sharp contrast that stands out to a discriminating eye. The second is when a scalp scar becomes visible due to the progression of MPHL.

\section{METHODS}

FUT, which is the technique of transplanting hair in its natural groupings of follicular units, was used exclusively in this study. In this technique, a horizontal strip of donor skin is taken from the back of the scalp and grafts are isolated under the microscope or grafts are individually harvested using follicular unit excision (FUE) methods. In our patients, incisions were made in the recipient area according to the natural direction and distribution of the patients' surrounding hairs using 18, 19, or $20 \mathrm{~g}$ solid needle trocars depending on the size of the grafts. SMP was used in one patient to camouflage the scalp scar by injecting dermal pigment into the scar and in the areas of MPHL. We used computerized tomography imaging (CAT scan) in one patient to locate a ventriculoperitoneal (VP) shunt before harvesting the donor skin strip.

\section{Case I}

A 27-year-old male patient who had a history of hydrocephalus during childhood and with an indwelling VP shunt was evaluated for MPHL with recession of temple hair exposing a sagittally oriented linear neurosurgical scar in the right fronto-parietal area (Figure 1). The patient was diagnosed with MPHL with extensive thinning in the front and crown areas of the scalp. During the first evaluation, he was found to have significant miniaturization (hair shafts were decreased in size, which is an indication of future progressive hair loss) in the Norwood Class VI pattern. The patient was initially started on finasteride (Propecia ${ }^{\circledR}$ ) in an attempt to stop or reverse his active hair loss. He was re-evaluated 21 months after his first visit. During this follow-up visit, the gross appearance was stable and there was no significant change seen in the trichoscopic evaluation. The patient was scheduled for hair transplant surgery using FUT to fill the scar and the surrounding areas of MPHL.

Prior to surgery, a CT scan of the patient's head was performed to assess the integrity of the skull and to determine the location of the VP shunt. The lower edge of the presumed strip incisions (where we would remove the skin containing donor hair) was marked with metal staples before imaging. The CT scan showed the location of the shunt in proximity but below the lower edge of the planned strip (Figure 2).

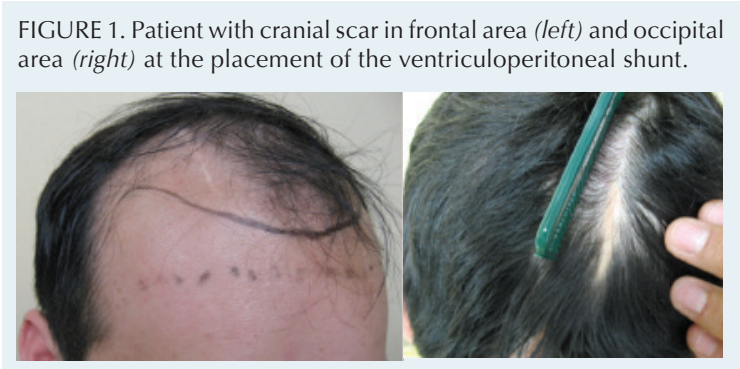

FIGURE 2. CT scan images with metal markers at the lowest level of presumed strip incision. Shunt is seen before exiting cranium.

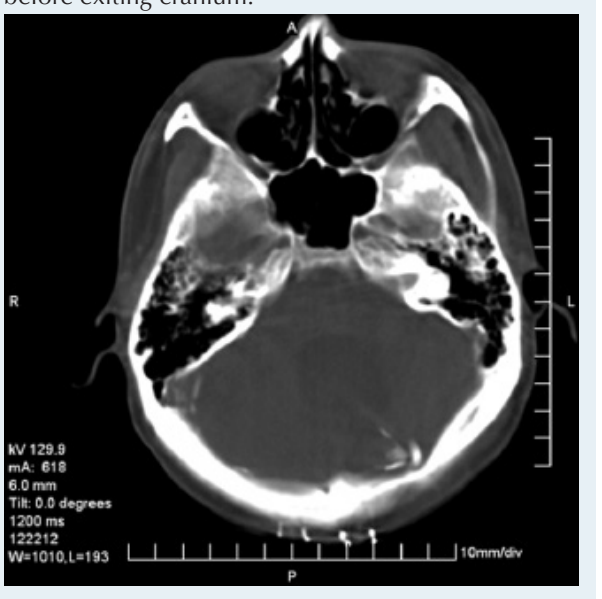


FIGURE 3. Strip is marked before donor excision. Note that the midline area of scalp is skipped due to its proximity to the ventriculoperitoneal shunt.

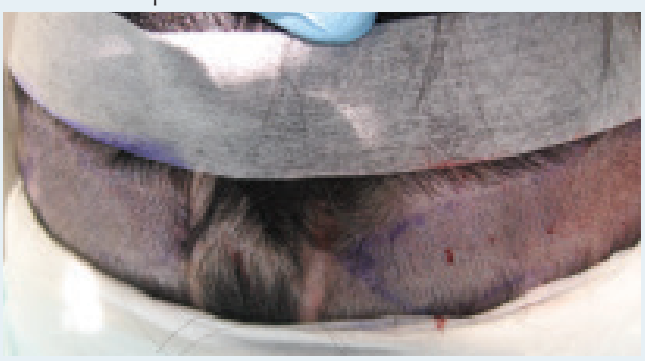

When harvesting the donor skin strip for hair transplantation, we avoided excising the skin in the area near the shunt hardware to prevent injury to the shunt (Figure

3). The donor wound was closed with absorbable sutures at the fascial level and staples at the skin level. After microscopic preparation of grafts, the frontal area of MPHL and scar were transplanted with 1,384 follicular unit grafts. (See Figure 4.) The patient was seen and evaluated several times in the first 2 weeks post-surgery as well as 7 months after his

FIGURE 4. Recipient area (left) and donor area (right) immediately after completion of hair transplant surgery and transplanting of 1,384 FU grafts.

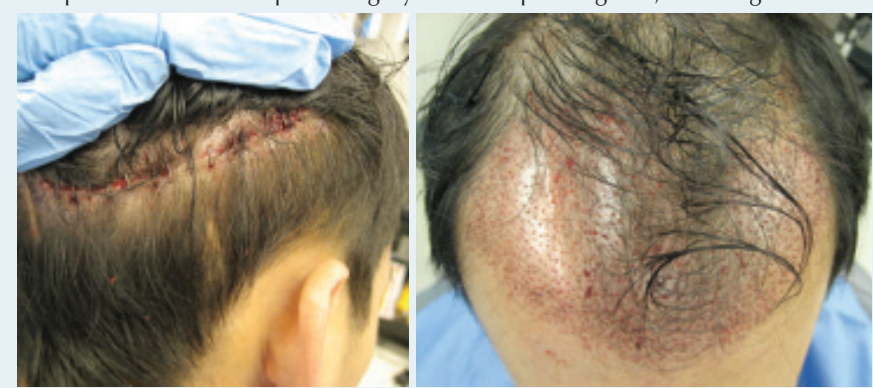

FIGURE 5. At 7-month follow-up, although the coverage is yet to be completed, scar is completely camouflaged.

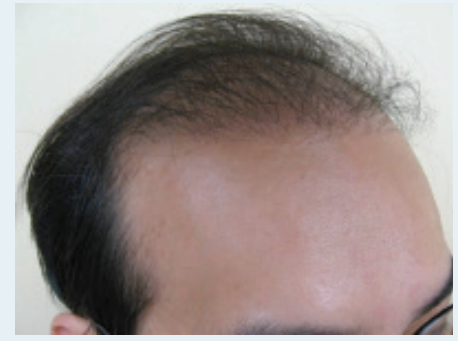

hair transplant procedure. The patient was satisfied with the coverage from his procedure because he could easily camouflage his neurosurgical scalp scar (Figure 5).

\section{Case II}

A 20-year-old male patient presented with a craniotomy scar about a half inch behind his original frontal hairline, which extended to the right temple. The proximity of the scar to the hairline made the scar visible. The patient had early MPHL with hairline and fronto-temporal triangle recession, which impaired the scar covering effect of his existing hair. (See Figure 6.) He was scheduled for a procedure to transplant grafts into the

FIGURE 6. Frontal scar of a cranial surgery (left) involving the entire frontal area with extension to the right temple area (right).

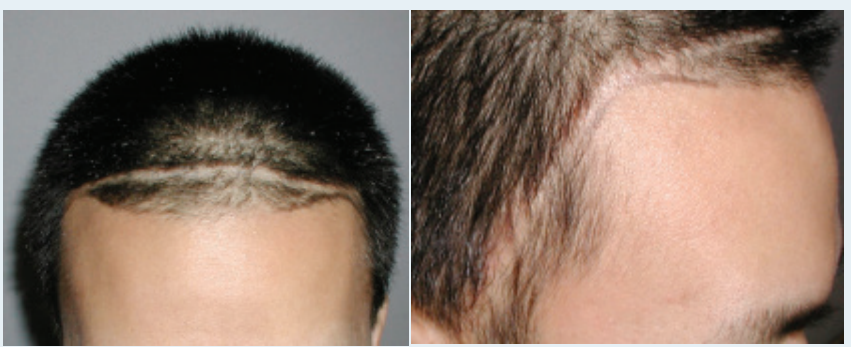

scar. During surgery in his frontal and temple areas, 519 follicular unit grafts were placed into the scar and surrounding areas

(Figure 7).

Although initially satisfied with the results, the patient returned to our clinic after four years with further recession of the hairline in

FIGURE 7. Immediate post-op; scar filled with 519 FU grafts.

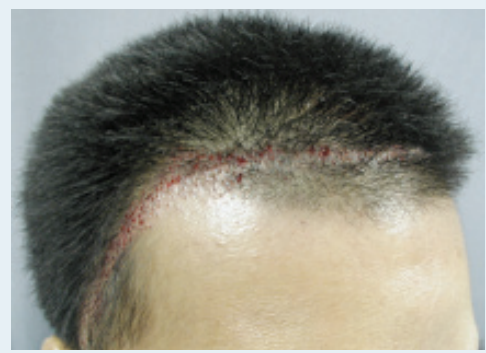

non-transplanted areas in the front and temple. The progression of his alopecia made the scar more noticeable in the front and corners. He subsequently underwent another hair transplant procedure of 1,021 follicular unit grafts for restoration of his hairline and frontal area and to add to the density in the scar that was transplanted four years earlier (Figure 8). The patient was seen in follow-up 6 months after his second surgery. He was pleased with the results and the scar was completely undetectable (Figure 9).

FIGURE 8. Before (left) and immediately after (right) surgery of frontal hairline restoration and reinforcement of scar area 4 years after first hair transplant procedure.
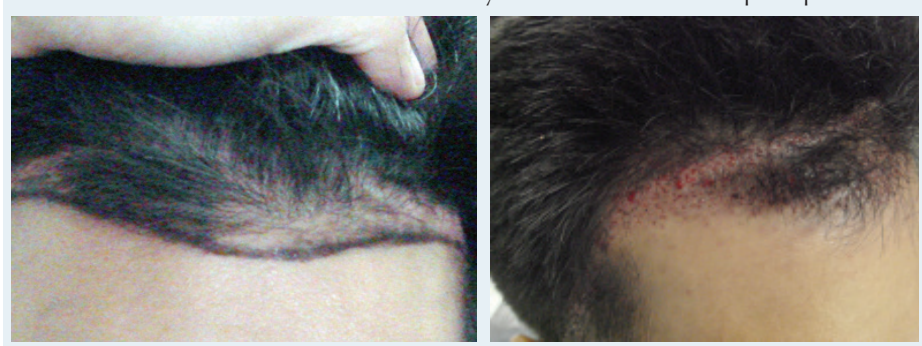

The goal of camouflaging the scar in this patient was achieved by filling the scar with grafts and increasing the density in the immediate areas of MPHL surrounding the scar.

\section{Case III}

A 36-year-old male patient presented with a large horseshoe cranial surgical scar, which measured be-

FIGURE 9. Six-month follow-up of second transplant consisting of 1,021 FU grafts in scar and frontal area.

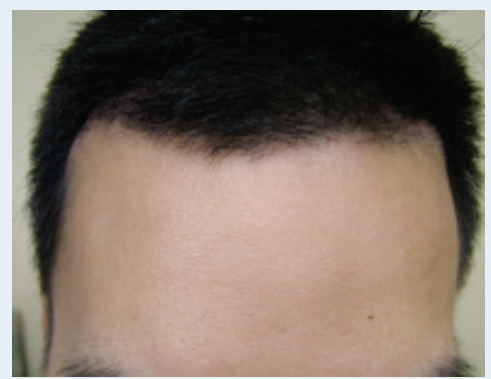

tween $1-2 \mathrm{~cm}$ in width, that extended from his left posterior parietal area to the occipital area (Figure 10). Five months before the patient's first visit with us, scar removal was attempted by a plastic surgeon but the scar showed no improvement. To produce enough density to cover the patient's

FIGURE 10. The 1-2cm-wide scar (left) extends from the left posterior parietal area to the occipital area (right).

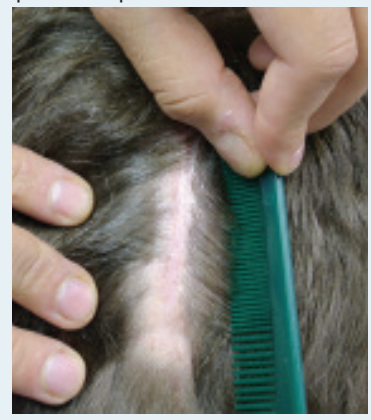

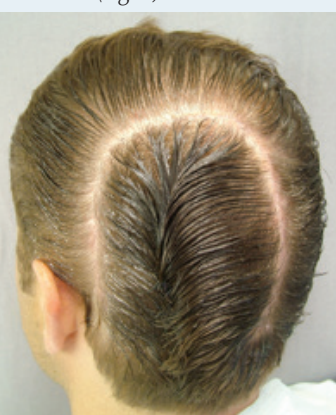


FIGURE 11. Immediate post-op (left) and at 8-month follow-up (right); $787 \mathrm{FU}$ grafts in scar.

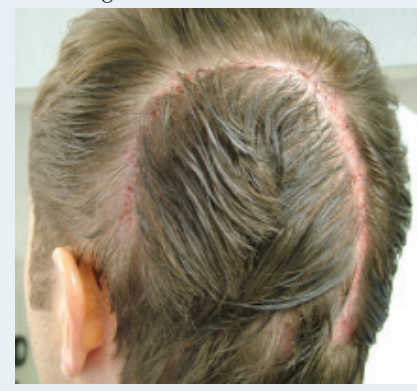

FIGURE 12. Thirty-one months post-op; a total of $1,595 \mathrm{FU}$ grafts transplanted into cranial scar.

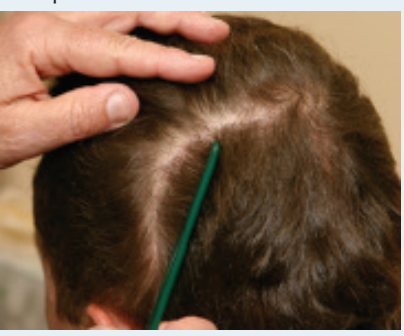

surgery, 808 FU grafts were transplanted. At follow-up afte 31 months, the patient's neurosurgical scar was noted to be completely camouflaged and undetectable (Figure 12).

\section{Case IV}

A 39-year-old male patient had FUT surgery using strip excision. He was evaluated for widening of his donor scar after completion of his healing at 9 months post-op. He opted for SMP and underwent 3 sessions, with one-week intervals between sessions, to add dermal pigments to the scar and areas of MPHL on the top and crown. Photos that were taken before and immediately after the third session of SMP are shown in Figure 13.

FIGURE 13. Before (left) and after (right) SMP procedure of a patient with previous strip hair transplant scar in the donor area.
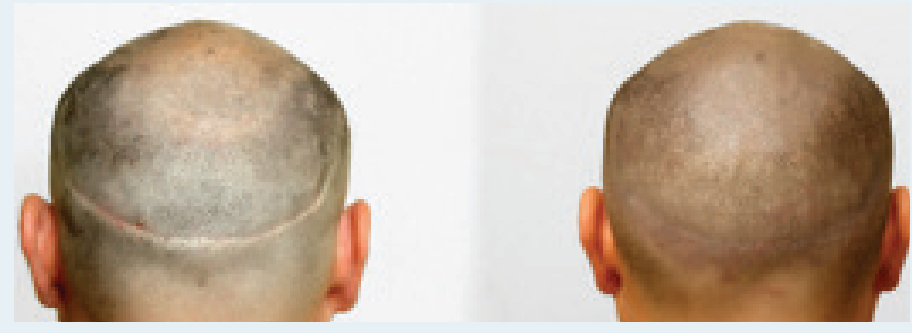

\section{DISCUSSION}

Scalp surgery can produce extensive scarring that cannot be disguised using typical styling methods and often this can be devastating for the patient. Although in many cases scalp scars can be hidden by a patient's existing hair, some scars in special anatomic locations cannot be hidden due to the inability of native hair to cover them. In patients who experience subsequent MPHL, the neurosurgical scar that has been initially hidden by the patient's existing hair may become detectable with the progression of MPHL.
New advancements in hair restoration procedures, particularly the emergence of FUT, have improved the appearance of transplanted hair. Hair transplantation can be used to camouflage disfiguring cranial scars. Many patients and physicians may be unaware of the possibility of using hair transplants to treat different types of scars when other methods cannot improve their appearance.. ${ }^{11,12}$ Based on the anatomic location of the scar, as well as the density of the surrounding hair and characteristics of the patient's native hair, one or more hair transplant procedures may be required to fill the scar with grafts and make it unnoticeable. SMP is another good method that can be used to camouflage or minimize the visibility of scars in selected patients.

\section{CONCLUSION}

Scars from cranial procedures or cranial trauma can cause significant aesthetic deformities for patients. While the undesirable scar might be detectable immediately after surgery, for half of the male population, it might not become visible until years after surgery as the genetic hair loss process progresses. Underlying scalp defects should be excluded preoperatively. A possibly impaired blood supply or atrophy in the scar may influence the surgical technique. Today's modern hair transplant surgery and SMP technologies offer these patients a way to mask a cranial scar by filling in the scar with grafts or dermal pigments, thus reducing or eliminating its visibility.

\section{References}

1. Iwami, K., et al. Cranial surgery without shaving: practice and results in our hospital. No Shinkei Geka. 2006(Sep); 34(1):901-905

2. Dvilevicius, A.E., et al. Craniotomy without trichotomy: analysis of 640 cases. Arq Neuropsiquiatr. 2004(Mar); 62(1):103-107.

3. Winston, K.R. Hair and neurosurgery. Neurosurg. 1992(Apr); 34(4):770.

4. Ratanalert, S., et al. Nonshaved cranial nrurosurgery. Surg Neurol. 1999(Apr); 51(4):458-463.

5. Miller, J.J., et al. Intracranial surgery: to shave or not to shave? Otol Neurotol. 2001(Nov); 22(6):908-911.

6. Marzola, M. Trichophytic closure of the donor area. Hair Transplant Forum Int'l. 2005; 15:113,116.

7. Frodel, J.L., and D. Mbrie. Optimal elective scalp incision design. Otolaryngol Head Neck Surg. 1999(Oct); 121(4):374-377.

8. Leach, P., et al. Zig-zag bicoronal scalp incision for cranio-facial cases in paediatric neurosurgery. Childs Nerv Syst. 2004(Jul); 20(7):483-484.

9. Rassman, W., et al. Scalp micropigmentation: a concealer for hair and scalp deformities. J Clin and Aesthetic Surg. 2015; 8(3):35-42

10. Bernstein, R.M., and W.R. Rassman WR. The logic of follicular unit transplantation. Dermatol Clin. 1999(Apr); 17(2):277-295.

11. Radwanski, H.N., et al. Follicular transplantation for the correction of various stigmas after rhytidoplasty. Aesthetic Plast Surg. 2007; 31(1):62-68.

12. Reed, M.L., and B.H. Grayson. Single-follicular-unit hair transplantation to correct cleft lip moustache alopecia. Cleft Palate Craniofac J. 2001(Sep); 38(5):538-540. 